\title{
Grasp Stiffness Control in Robotic Hands through Coordinated Optimization of Pose and Joint Stiffness
}

\author{
Virginia Ruiz Garate ${ }^{1}$, Maria Pozzi ${ }^{2}$, Domenico Prattichizzo ${ }^{2}$, Nikos Tsagarakis ${ }^{3}$, and Arash Ajoudani ${ }^{1}$
}

\begin{abstract}
This work presents a novel controller for robotic hands that regulates the grasp stiffness by manipulating the pose and the finger joint stiffness of hands with multiple degrees of freedom while guaranteeing the grasp stability. The proposed approach is inspired by the observations in human motor behaviour that reveal a coordinated pattern of stiffening among the hand fingers, along with a predictive selection of the hand pose to achieve a reliable grasp. The first adjusts the magnitude of the grasp stiffness, while the latter manipulates its overall geometry (shape). The realization of a similar control approach in robotic hands can result in a reduction of the software complexity and also promote a novel mechanical design approach, in which the finger stiffness profiles of the hand are adjusted by only one active component. The proposed control is validated with the fully actuated Allegro Hand, while trying to achieve pre-defined grasp stiffness profiles or modifications of an initial one.
\end{abstract}

Index Terms-Grasping, Multifingered Hands, Stiffness Control, Underactuation

\section{INTRODUCTION AND RELATED WORK}

$\mathbf{R}$ OBOTIC hands with multiple degrees of freedom (DoF) offer the high level of dexterity required to perform complex manipulation tasks [1]. Such advantage, however, is accompanied by a raised level of complexity in the control architecture to guarantee both the stability of the grasp and the versatility of the operation [2].

Force control is one of the most well-known techniques to achieve such reliable grasps while manipulating objects or interacting with the external world [3]. Alternatively, the grasp forces can be indirectly modulated through grasp compliance control. The latter presents an enhanced stability to unexpected changes of the environment due to the dominance of the compliance term in the dynamic response [3]-[5]. Impedance

Manuscript received: February, 24, 2018; Revised May, 26, 2018; Accepted July, 16, 2018.

This paper was recommended for publication by Editor Han Ding upon evaluation of the Associate Editor and Reviewers' comments. This work was supported in part by the EU H2020 projects "SOMA: SOft MAnipulation" (no. 645599), and "SoftPro" (no. 688857)

${ }^{1}$ Virginia Ruiz Garate and Arash Ajoudani are with the HumanRobot Interfaces and Physical Interaction Research Line, Istituto Italiano di Tecnologia, Genova, Italy. virginia.ruizeiit.it, arash.ajoudani@it.it

${ }^{2}$ Maria Pozzi and Domenico Prattichizzo are with the Department of Information Engineering and Mathematics, University of Siena, Italy and the Advanced Robotics Department, Istituto Italiano di Tecnologia, Genova, Italy. pozzi@diism.unisi.it, prattichizzo@ing.unisi.it

${ }^{3}$ Nikos Tsagarakis is with the Human-Robot Interfaces and Physical Interaction Research Line, Istituto Italiano di Tecnologia, Genova, Italy. nikos.tsagarakis@iit.it

Digital Object Identifier (DOI): see top of this page. control has also been studied for dual-arm manipulation [6]. In fact, it could be considered an alternative form of hand grasp, in which each robotic arm corresponds to a finger but without sharing the base. Still, the applicability of force or impedance control approaches at large scale can be limited by the need for accurate torque sensing and tracking in finger joints, usually associated with a high cost. This has been the main motivation for the design of underactuated hands with active impedance [7], or the ones with coupled, passive elastic joints [8].

On the other hand, the complex biomechanical architecture of the human hand raises challenging questions for understanding the control strategies that underlie the coordination of movements and forces required for a wide variety of tasks. These can range from the individual movements of single joints to multi-DoF in-hand manipulation [9], [10]. The problem of redundancy in kinematic control of the hand has been tackled by reducing the state space of many dimensions to a control space of few, commonly known as the postural hand synergies [11]. Embedding such coordinated movements in the joint space has shown promising results, either using software [12] or hardware [7] solutions.

Likewise, one of the first attempts to explore the concept of human hand synergies in the stiffness coordinates investigated the existence of a coordinated stiffening pattern in human fingertips during a tripod grasp [13]. In this study, the human fingertip stiffness profiles are estimated using external stochastic perturbations and illustrated by ellipsoids. The preliminary results of this study suggest that the co-activations of the forearm muscles contribute to a coordinated stiffening of the fingers. This leads to an increase in the amplitudes of the major axes of the ellipsoids with minor effects on their orientations. Still, effective modulations of the reaction forces in arbitrary directions call for the ability to modify the overall geometry of the grasp stiffness ellipsoid [14]. For example, while using a carving tool, surface deformations can be treated with different interaction force profiles, the behaviour of which is described by the tool tip (Cartesian) stiffness ellipsoid geometry. More precisely, e.g., when drilling a wall, it is required to be very stiff in the direction perpendicular to the surface, while remaining compliant in the others to adapt to possible external disturbances.

To modify the endpoint stiffness geometry, humans explore the dominant role of self-selected configurations [15]. This can be explained by analysing the roles of Jacobians in the conservative congruence transformations from the muscle stiffness to the joint space, and consequently to the endpoint 
[16], [17]. Also, static postures are more energy-efficient solutions in comparison to co-contractions [15].

Towards exploring the relevance of this concept to robotic applications, the work in [18] implements a similar control strategy for the teleoperation of a redundant robotic arm. The volume-adjusting component of the endpoint stiffness is achieved by using the Common-Mode Stiffness (CMS) variable. CMS implements a coordinated activation across the arm joints. The control of redundant kinematic DoF is achieved simultaneously using a Configuration-Dependent Stiffness (CDS) variable. CDS controls the nullspace velocity of the manipulator to change the overall geometry of the Cartesian stiffness ellipsoid.

Therefore, a combined CDS/CMS principle can be used to realize variable stiffness articulated hands with $n+1$ actuation, being $n$ degrees of freedom used for the robot position control, and only one to implement the CommonMode Stiffness (e.g. by changing the pre-tension of the joint springs in a coordinated way). In the case that active stiffness regulation of the individual finger joints is possible by the actuation system, the generation of joint stiffness references values can be simplified by the consideration of the synergistic stiffness regulation principles. In this way the robotic hand control references would be simplified from controlling $2 \times \mathrm{n}$ parameters to only $n+1$.

The work presented here aims to exploit these concepts of CDS and CMS to regulate the grasp stiffness of a robotic hand while assuring its stability. This approach differs from the one in [19] first from the problem definition perspective. Being able to represent the grasp stiffness by an ellipsoid, the method proposed here focuses on reproducing its main geometrical features, instead of the overall stiffness matrix numerical values targeted before. Moreover, from the methodology point of view, previous method provided a first exploration of all feasible grasp configurations keeping same contact points. This outputted a CDS base. Then, a nullspace exploration was done to locally optimize the solution. At last, the joint stiffness was optimized (CMS) to minimize magnitude differences. The current approach, instead, provides full joint trajectories based on a simultaneous optimization of the hand pose and joints stiffness (CDS/CMS). Additionally, it ensures contact stability throughout the trajectory, whereas previously stability was only checked at the end of the process. Finally, before only spherical objects where tested in a simulation environment. The new proposed approach is further evaluated through an experimental set-up with the 16-DoF Allegro Hand [20], and using a wider range of objects.

\section{GRASP STIFFNESS PROBLEM DEFINITION}

When holding an object, the grasp stiffness matrix $\mathbf{K} \in$ $\mathbb{R}^{6 \times 6}$ relates the wrench $\Delta \mathbf{w} \in \mathbb{R}^{6}$ applied to the object to its displacement $\Delta \mathbf{u} \in \mathbb{R}^{6}: \Delta \mathbf{w}=\mathbf{K} \Delta \mathbf{u}=\left(\mathbf{G K}_{c} \mathbf{G}^{T}\right) \Delta \mathbf{u}$, where $\mathbf{K}_{c}$ is the equivalent stiffness matrix at the contact points taking into account all the system compliance sources [21]. $\mathbf{G}$ is the grasp matrix relating the contact forces and moments transmitted through the contact points, to the set of wrenches that the hand applies on the object [22], [23].
The stiffness matrix $\mathbf{K}_{c}$ incorporates the fingers and object structural elasticity [23]:

$$
\mathbf{K}_{c}=\left(\mathbf{C}_{s}+\mathbf{J K}_{q}^{-1} \mathbf{J}^{T}\right)^{-1},
$$

where $\mathbf{C}_{s}$ represents the structural compliance matrix, $\mathbf{K}_{q}$ is a $n_{q} \times n_{q}$ diagonal matrix representing the joint stiffness, and $\mathbf{J}$ is the hand Jacobian matrix. $n_{q}$ is the total number of finger joints in the hand ${ }^{1}$.

The objective of the presented method is to reach a desired grasp stiffness, namely $\mathbf{K}_{d}$ ), from a different initial one $\mathbf{K}$. This is achieved by means of a simultaneous control of the hand pose $\mathbf{q}=\left[q_{1}, q_{2}, \ldots q_{n q}\right]$, and the joint stiffness $\mathbf{K}_{q}$. The adaptation of the pose $\mathbf{q}$ implements the CDS control and contributes to the geometrical variations of the grasp stiffness. $\mathbf{K}_{q}$ is based on the synergistic finger stiffness concept (CMS) and therefore mainly contributes to the modifications of the stiffness ellipsoid volume. For every finger $f$, this joint stiffness is a diagonal matrix $\mathbf{K}_{q, f}=\alpha \boldsymbol{\Gamma}_{f}$, with $\mathbf{K}_{q, f} \in \mathbb{R}^{n q_{f} \times n q_{f}}$, where $n q_{f}$ is the number of joints of the finger, $\alpha$ is the common mode stiffness parameter $(\mathrm{Nm} / \mathrm{rad})$, and $\boldsymbol{\Gamma}_{f}$ is a constant normalised vector implementing the coordinated stiffening of the hand fingers [13]. Therefore, the maximum achievable grasp stiffness volume is limited by the maximum applicable $\alpha$.

The algorithm focuses on the translational part of the desired stiffness $\left(\mathbf{K}_{d}\right)$, referred to as $\mathbf{K}_{t, d}$. Being the translational part of the stiffness representable by a $3 \mathrm{D}$ ellipsoid, the method aims to match the geometrical features of $\mathbf{K}_{t, d}$. These geometrical features are defined by the ellipsoid's 3 principal axes and the relationship between them. As targeting all the possible parameters would render the objective function too sparse, the method focuses on the two main axes of $\mathbf{K}_{t, d}$, targeting, in descending order of importance: (i) main axis orientation of the grasp stiffness, (ii) secondary axis of the grasp stiffness, and (iii) length ratio of the two main axes. In this way, the desired stiffness geometry is emphasized, providing a high level description of the task requirements. The stiffness magnitude can be adjusted to maintain the stability or based on the sensory feedback.

The values previously listed are obtained from the SVD decomposition of $\mathbf{K}_{t}$. The difference between two axis (desired $d$ and actual one $i$ ) is defined as: $\theta=\arccos \left(\mathbf{U}_{d}^{T} \mathbf{U}_{i}\right)$, where $\mathbf{U}$ is the corresponding axis unitary vector (main or secondary). Because there can be two possible angles defined between both axes, the absolute smallest one is kept between the previous and $\theta=\arccos \left(-\mathbf{U}_{d}^{T} \mathbf{U}_{i}\right)$. On the other hand, the error in length ratio of the two main axes is defined as $\beta=\left|\frac{D_{d, 1}}{D_{d, 2}}-\frac{D_{i, 1}}{D_{i, 2}}\right| . D_{1}$ stands for the main eigenvalue and $D_{2}$ for the secondary one.

A grasp quality criteria is also defined following the method in [24]. The index called Potential Contact Robustness (PCR) is based on the distance of the contact force to the friction cone, increasing as the grasp becomes more robust. We adjust the general equation to focus only on the Coulomb friction constraint and the maximum distance to the friction cone [24].

\footnotetext{
${ }^{1}$ In this work only finger joint movements are considered, keeping a fixed wrist configuration.
} 
For every contact point $c$, it is stable if: $\epsilon\left\|\boldsymbol{\lambda}_{c}\right\|-\boldsymbol{\lambda}_{n, c}<s$, where $\boldsymbol{\lambda}_{c}$ represents the contact forces and $\boldsymbol{\lambda}_{n, c}$ the normal component of those forces. $\epsilon=\frac{1}{\sqrt{1+\mu^{2}}}$ being $\mu$ the coefficient of friction, and $s$ a security margin.

\section{METHODOLOGY}

The proposed method consists of two main consecutive steps: (i) definition and stabilization of an initial grasp, (ii) optimization of the grasp towards the desired stiffness.

It must be pointed out that, due to the lack of sensory input, this method mainly relies on a hand model provided in terms of Denavit-Hartenberg parameters and finger dimensions. The hand is equipped with joint position encoders and torque control through current sensing. As no visual information is available during the optimization process, the new object locations need to be estimated (see Section III-A4). Grasp stiffness is computed by using the equations of Section II. Also, no fingertip force sensors are available, so it is not possible to directly detect the contact points. Hence, these are calculated from the stabilized hand model and the estimated object location (see Section III-A3).

During the following description of the method we will refer to two parallel hands and objects:

- Real hand and real object: the actual robotic Allegro Hand and object used.

- Virtual hand and virtual object: the models created by software from the mechanical parameters and dimensions of the real ones.

\section{A. Initial grasp}

To define an initial grasp the following steps are followed:

1) User initial grasp: The hand is first set to a predefined configuration with low stiffness, mainly in gravity compensation. From here a manual or automated grasp is defined. Because of the lack of sensory information, objects are generalized as spheres or cuboids. Then, the encoder values of the real hand are measured and a virtual model of the hand and the object in the initial grasp is created.

2) Definition of initial contact points: From the obtained hand and object virtual models, the contact points between both are defined. So far we consider only fingertip grasps. As no force sensor is provided, and due to the limitations in terms of encoder accuracy and possible irregularities in the object surface, the virtual hand might not always be exactly in contact with the virtual object. Therefore, contact locations are approximated to be exactly at the fingertips, which positions can be retrieved from direct kinematics. All contacts are modelled as hard finger contacts, where only forces (and no moments) are transmitted [22].

3) Stabilization of the initial grasp: The objective of this step is to find the joint reference positions such that the hand squeezes the object and stabilizes the initial grasp. To do so, an optimization is carried out where a cost function based on the PCR index is computed using the method in [25]. This optimization computes a set of internal forces such that the contact forces $\boldsymbol{\lambda}_{c}=-\mathbf{G}^{\#} \mathbf{w}+\mathbf{E} \mathbf{y}$ are as far as possible from their friction cone limits. $w$ stands for the external

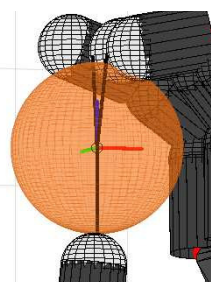

(a)

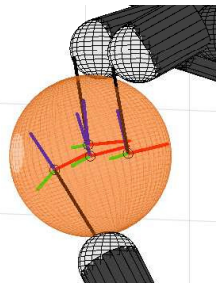

(b)
Fig. 1: Extra links (black lines) defined to obtain the virtual object center (a) in a initial configuration (all centred) (b) at the end of an optimization. Each fingertip is linked to a corresponding object location. The mean of all of them provides the computed new center.

forces applied and $\mathbf{E}$ represents the basis of the controllable subspace of internal forces. $\mathbf{y}$ is a vector that parametrizes the homogeneous part of the solution of the equation $\mathbf{w}=-\mathbf{G} \boldsymbol{\lambda}_{c}$. Once an optimal set of internal forces $\boldsymbol{\lambda}_{o p t}=\mathbf{E y}$ is found, the displacement of the end-effector (fingertips) corresponding to that force can be retrieved as $d \mathbf{x}=\mathbf{K}_{c}^{-1} \boldsymbol{\lambda}_{\text {opt }}$. The complementary joint displacement $d \mathbf{q}=\mathbf{J}^{T}\left(\mathbf{J J}^{T}\right)^{-1} d \mathbf{x}$ is then applied to the virtual hand model, while keeping the virtual object in place.

As a general procedure (now and during the following grasp optimization), to actually check if the virtual hand is in contact with the object as desired, contacts between the virtual stabilized hand and the virtual object are searched for. As the stabilized virtual hand position should drive the fingers towards the inside of the object, the contact points must be detectable. If they are not found, then for sure the contact has been lost. These contact points are obtained analytically as the intersection between the fingertips of the stabilized virtual hand, modelled as spheres of a known radius $r_{s}$, and the virtual object.

A final double check is performed to assure that the newly found configuration is stable. For this, the method described at the end of Section II is used being $\boldsymbol{\lambda}_{\text {opt }}=\mathbf{E y}$. If the grasp proves to be stable, this configuration is finally commanded to the real hand, assuring the stable initial grasp.

4) Estimation of the motion of the virtual object: Again, due to the lack of sensory information, it is not possible to know at each step where exactly the object is, except on the initial position. Therefore, the virtual object position is approximated as the mean of the possible locations retrieved from each finger. To do this, an additional virtual spherical joint at the fingertip of each contacting finger is created. This spherical joint connects the fingertip to the object center frame by means of a virtual link. Fig. 1a shows an example of the initial definition of these virtual links. The length of this link is defined from the initial grasp configuration. At this initial position, from each fingertip reference frame with respect to the world frame ${ }^{w} \mathbf{T}_{f_{t}}$, the transformation matrix from the fingertip to the object center can be obtained ${ }^{f_{t}} \mathbf{T}_{o}={ }^{w} \mathbf{T}_{f_{t}}^{-1}{ }^{w} \mathbf{T}_{o}$. From this matrix, the set of roll, pitch, and yaw rotations for the spherical joint can be obtained. Likewise, during the optimization process, using a new set of finger joint positions, spherical joint positions, and the initial virtual links length, the new virtual object center can be estimated. 


\section{B. Grasp stiffness optimization}

Once the initial grasp is defined, the main optimization starts. The optimization is repeated to a maximum number of iterations and is defined as:

$$
\begin{array}{ll}
\underset{\mathbf{x}}{\operatorname{minimize}} & \mathrm{f}(\mathbf{x})=h_{1} K_{e}+h_{2} \sum\left(\Delta f_{t}\right)+\frac{1}{P C R} \\
\text { subject to } & \mathbf{x}_{\min } \leq \mathbf{x} \leq \mathbf{x}_{\max }
\end{array}
$$

being $\mathbf{x}=\left[\dot{q}_{x}, \alpha\right]$, where $\dot{q}_{x}$ is the vector of joint angular velocities enlarged with the additional spherical joint angular velocities at the fingertips (needed to find the virtual object center), and $\alpha$ is the synergistic value of joint stiffness in $\mathrm{Nm} / \mathrm{rad} . K_{e}$ is itself defined as a weighted function of the difference between the desired and actual stiffness based on the parameters described in Section II:

$$
K_{e}=\theta_{1}+0.5 \theta_{2}+0.01\left|\frac{D_{d, 1}}{D_{d, 2}}-\frac{D_{i, 1}}{D_{i, 2}}\right|,
$$

where $\theta_{1}$ and $\theta_{2}$ represent the error in the main and secondary axes orientation respectively. $\sum\left(\Delta f_{t}\right)$ stands for the sum of the difference between the initial position of the fingertips with respect to the object frame ${ }^{o} \mathbf{P}_{f_{t}, 0}$, and the actual one ${ }^{o} \mathbf{P}_{f_{t}, i}$ : $\Delta f_{t}=\left\|{ }^{o} \mathbf{P}_{f_{t}, i}-{ }^{o} \mathbf{P}_{f_{t}, 0}\right\|$. This term tries to minimize the movement of the fingertips with respect to the object center and consequently, to keep a constant position of the contact points. As the fingertips are allowed to rotate around the contact point and because of possible inaccuracies between the virtual models and the real hand/object, a movement threshold is established depending on the hand design. ${ }^{o} \mathbf{P}_{f_{t}}$ can be defined as the translation vector of the transformation matrix ${ }^{o} \mathbf{T}_{f_{t}}={ }^{w} \mathbf{T}_{o}{ }^{w} \mathbf{T}_{f_{t}}$, where ${ }^{w} \mathbf{T}_{f_{t}}$ is the transformation matrix from the world frame to the fingertips obtained from direct kinematics. To determine $h_{1}$ and $h_{2}$ in (3), several weights were examined in various simulations. $h_{1}=10^{6}$ and $h_{2}=10^{10}$ were found to work best for several defined stiffness while imposing the needed constraint on the fingertip movement.

For each iteration, the set of new joint positions is obtained as $\mathbf{q}_{x, k+1}=\mathbf{q}_{x, k}+\dot{\mathbf{q}}_{x, k} \Delta t$. The new hand poses provide the CDS control modelling the overall stiffness geometry (shape). Using direct kinematics the new fingertip position can be obtained, and using the new spherical joint positions the current object frame from each fingertip is computed (see Section III-A4). The new virtual object frame is established as the mean value of those obtained from each contacting finger (see as an example Fig. 1b).

Then, following the same stabilizing procedure as in Section III-A3, a virtual hand pose that stabilizes the new grasp is searched. This stabilization also depends on the new joint stiffness $\alpha$. As introduced, $\alpha$ mainly manipulates the volume of the ellipsoid providing the CMS control. Therefore, if the found value is less than the initial one, the latter is kept to preserve a similar overall magnitude of the stiffness. In the end, depending on the task specifications, the user can increase/decrease this value up to the maximum allowed or down to the minimum specified by the optimization.

The corresponding new contact points, grasp matrix, hand Jacobian, and optimization function output are then computed. (a)

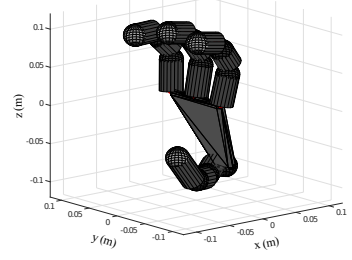

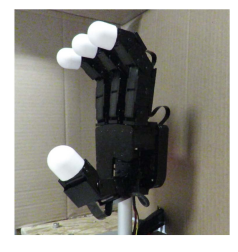

(b)

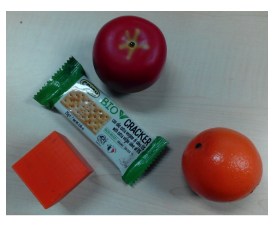

(c)
Fig. 2: (a) Model of the Allegro Hand implemented in Syngrasp in the home position (b) Allegro Hand in home position (c) Set of tested objects.

Finally, at each iteration, for the newly found configuration the following conditions are verified:

- the new joint positions do not exceed the joint limits,

- the hand actually moved, i.e., at least one of the joints moved more than $0.01 \mathrm{deg}$,

- the contact points did not move more than the specified threshold,

- no contact point has been lost,

- no contact point becomes unstable.

If all the conditions are satisfied, then the real and virtual hands are moved to the newly found stable position and the values are stored. Otherwise, the algorithm concludes.

\section{EXPERIMENTAL VALIDATION}

We implemented the algorithm in MATLAB 2013a (The MathWorks Inc.) with the Syngrasp and Robotics toolboxes [26], [27]. We tested our control strategy on the fully actuated Allegro Hand [20]. Fig. $2 a$ and $2 b$ show the virtual and real hand in a known "home" position. For the communication with the real hand, the ROS library from Matlab is used.

\section{A. Definition of parameters}

For the grasp definition, the structural compliance in (1) $\mathbf{C}_{s}$ is set to a generic value of $10^{-4} \mathrm{~m} / \mathrm{N}$, signifying stiff finger contacts. The joint stiffness synergy is defined as $\boldsymbol{\Gamma}_{f}=$ $[0.7,0.95,1,0.8]$ for the index, middle, and little fingers, and $\boldsymbol{\Gamma}_{f}=[1,0.95,1,0.95]$ for the thumb. These values are chosen based on the mechanical characteristics of the hand and the chosen vertical set-up (see Fig. 2b). An initial synergistic value $\alpha$ of $4 \mathrm{Nm} / \mathrm{rad}$ is chosen.

For the stability optimization described in Section III-A3, a minimum and maximum contact force of 2 and $5 \mathrm{~N}$ respectively are specified. This avoids getting a reference position that goes too much inside the object which, due to the hand configuration, would provide a big push from the upper fingers and prevent the lower finger (the thumb in this case) from moving. For the contact stability check, the coefficient of friction is set to $\mu=0.8$, and the security margin $s$ to -0.01 .

The stiffness optimization is repeated for a maximum of 25 iterations with step variable of $\Delta t=0.1 \mathrm{~s}$. A maximum and minimum joint velocity of $\dot{\mathbf{q}}= \pm 0.52 \mathrm{rad} / \mathrm{s}( \pm 30 \mathrm{deg} / \mathrm{sec})$ is set, while $\alpha$ is constrained between 2 and $6 \mathrm{Nm} / \mathrm{rad}$. The joint angular velocities are initialized with a random value between 0 and $0.52 \mathrm{rad} / \mathrm{sec}$. 
At each step, the process only continues if $\Delta f_{t}=\|{ }^{o} \mathbf{P}_{f_{t}, i}-$ ${ }^{o} \mathbf{P}_{f_{t}, i-1} \|<3 \mathrm{~mm}$. An extra check is done to be sure that in general the accumulated displacement does not increase above $5 \mathrm{~mm}$ from the initial position. These threshold values are chosen based on the Allegro Hand fingertip dimensions to allow a certain movement around the initial contact point. We tested a 3 fingertip grasp, though the method is adaptable for grasps with more/less contacts.

\section{B. Impedance control}

The Allegro Hand is fully actuated and controlled in torque. Therefore an impedance controller is implemented based on the desired joint positions $\left(\mathbf{q}_{d}\right)$ and stiffness: $\boldsymbol{\tau}_{d}=\mathbf{k}_{q}\left(\mathbf{q}_{d}-\right.$ $\mathbf{q})-\mathbf{k}_{d} \dot{\mathbf{q}}$, where $\mathbf{k}_{q}=\operatorname{diag}\left(\mathbf{K}_{q}\right)$ is the desired joint stiffness, and $\mathbf{k}_{d}$ is the corresponding damping found from already stable coefficients $\mathbf{c}_{f}$ as $\mathbf{k}_{d}=\sqrt{\frac{\mathbf{k}_{q}}{\mathbf{c}_{f}}}$. To the desired torque value $\tau_{d}$, an additional term is added to compensate for the gravity: $\boldsymbol{\tau}=\boldsymbol{\tau}_{d}+\boldsymbol{\tau}_{g}$. This gravity compensation is provided by the software distributed with the hand.

For each iteration, when the desired new configuration is sent to the hand, the movement is smoothed by driving it in a 5 step linear trajectory.

\section{Definition of experimental tests}

The proposed method is tested with the 4 different objects ( 2 spheres and 2 cuboids) depicted in Fig. 2c. The objective stiffness can be defined from geometric modifications of the initial one, or by manually setting the values depending on the task (e.g., in a placing task where grasp stiffness should be very high in the entering direction and quite compliant in the others). First, three different sets of modifications are considered for the same object (the apple). Then, to demonstrate that our method can be applied to different objects, one test is presented for each of them.

For the apple the following cases are chosen to show the different convergence features of the method:

- From an initial extended apple grasp, rotate the initial stiffness $\operatorname{Rot}\left(Y, \frac{\pi}{4}\right)$,

- from the same initial grasp stiffness, rotate it $\operatorname{Rot}\left(Z, \frac{\pi}{4}\right)$,

- from a different initial folded apple grasp, rotate the initial stiffness $\operatorname{Rot}\left(Y, \frac{-\pi}{4}\right)$.

As the first and last experiment with the apple are opposite to each other, it is forecasted that the hand configuration and stiffness results will be complementary (initial of first experiment similar to final of third and viceversa).

For the cube, from an initial folded grasp position, a desired diagonal stiffness is targeted such that the grasp would be most stiff in the ' $\mathrm{X}$ ' direction: $\mathbf{K}_{t, d}=\operatorname{diag}(2000,100,200)$ $\mathrm{N} / \mathrm{m}$. Same stiffness is targeted from a folded grasp with the orange. As both objects have similar dimensions, it is forecasted that the resulting movement should be similar. In case of the pack of "crackers", from a middle positioned initial grasp, a different desired diagonal stiffness is targeted such that the grasp would be most stiff in the ' $\mathrm{Z}$ ' direction: $\mathbf{K}_{t, d}=\operatorname{diag}(200,100,2000) \mathrm{N} / \mathrm{m}$.

The following results are reported in numerical tables:
- Initial and final error on the main $\left(\theta_{1}\right)$ and secondary axes $\left(\theta_{2}\right)$ of the stiffness,

- length ratio error of the two main axes $\beta$,

- final minimum $\alpha$ value,

- initial and final PCR values.

Furthermore, to prove the usefulness of the grasp robustness term (PCR) and stabilization procedure (Section III-A3), we run an alternative optimization in which the stabilization is not taken into account. To do so, starting from the initial stable grasp (Section III-A3), we remove the stabilization process in the subsequent optimized hand poses. Also, the optimization problem defined in (2) is adapted by deleting the robustness term (PCR). To compare both methods in terms of stability, it is observed if the hand is able to maintain the object grasp during the stiffness manipulation and consequent changes in the hand pose. 20 repetitions of each case are performed for each of the methods.

\section{Experimental results and discussion}

Fig. 3 to Fig. 5 show the results for the three apple cases. The sub-figures corresponding to the real and virtual hands of the second case were omitted for the sake of space, as the initial configuration was similar to the first case. Nonetheless, several repetitions of all the experimental cases can be seen in the multimedia extension at [28]. Please note that the virtual hands correspond to the stabilized model, i.e., the one in which the fingers are projected slightly inside the object. Numerical results for all tests are displayed in Table I. The video at [28] shows an example of stiffness manipulation corresponding to the experiment in Fig. 3.

TABLE I: Initial values and results of the algorithm when manipulating the apple-like object. $\theta$ error is expressed in $\operatorname{deg}, \beta$ and PCR are dimensionless, and $\alpha$ is $N m / r a d$. 'Init' stands for initial configuration.

\begin{tabular}{|c|c|c|c|c|c|}
\hline Apple & $\boldsymbol{\theta}_{\mathbf{1}}$ & $\boldsymbol{\theta}_{\mathbf{2}}$ & $\boldsymbol{\beta}$ & $\boldsymbol{\alpha}$ & PCR \\
\hline Init $\left(Y, \frac{\pi}{4}\right)$ & 44.81 & 44.94 & 0 & 4 & 0.1288 \\
\hline Final $\left(Y, \frac{\pi}{4}\right)$ & 9.676 & 29.25 & 0.1304 & 2.513 & 0.1397 \\
\hline Init $\left(Z, \frac{\pi}{4}\right)$ & 38.25 & 23.16 & 0 & 4 & 0.1288 \\
\hline Final $\left(Z, \frac{\pi}{4}\right)$ & 18.5 & 10.24 & 0.0108 & 3.379 & 0.1351 \\
\hline Init $\left(Y, \frac{-\pi}{4}\right)$ & 45 & 44.89 & 0 & 4 & 0.1647 \\
\hline Final $\left(Y, \frac{-\pi}{4}\right)$ & 14.29 & 8.616 & 0.05732 & 6 & 0.1307 \\
\hline
\end{tabular}

An overall decrease of the grasp stiffness orientation error is observed. However, the perfect final orientation for both main axes is not reached. To be able to have no error, the desired stiffness should be defined from values known to be achievable for the considered hand. The definition of such a stiffness would require a deep study on the large set of possible grasp configurations and graspable objects by each evaluated hand. Nonetheless, in all cases the orientation error is significantly reduced, as can be observed from the ellipsoid shapes (Fig. $3 \mathrm{c}, 4$, and 5c). The worst case (second case) reaches an error reduction for the main orientation of almost $50 \%$, and more than $50 \%$ for the secondary axes. In this case, the largest desired stiffness is in the transversal ' $\mathrm{XYZ}$ ' direction, translating in an horizontal movement of the fingers (in ' $\mathrm{XY}$ ' plane), which is the most constrained one. Another noticeable point is that, as predicted, in the third experiment, starting 


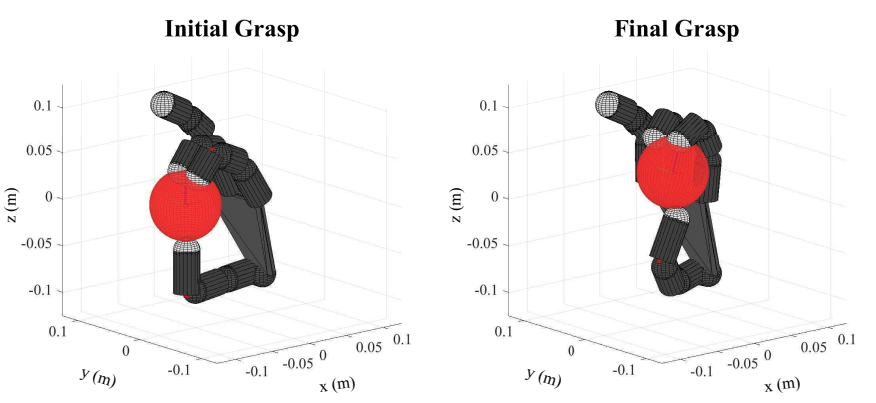

(a)

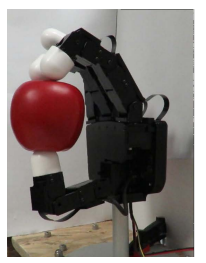

(b)

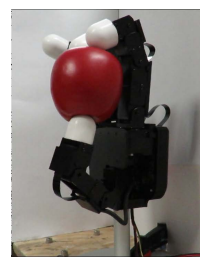

Initial Stiffness

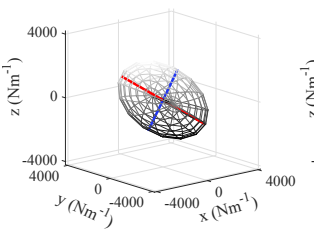

Objective Stiffness

Final Stiffness

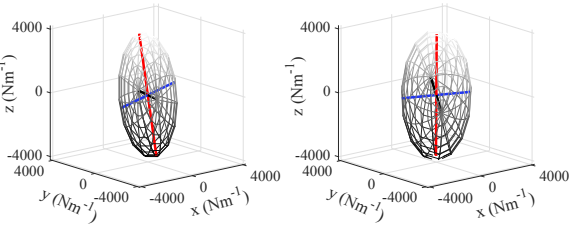

(c)

Fig. 3: First apple experiment $\left(\operatorname{Rot}\left(Y, \frac{\pi}{4}\right)\right)$. (a) Stabilized model of the Allegro Hand in the initial and final configurations (b) Allegro Hand in initial and final configurations (c) $\mathbf{K}_{t}$ initial, targeted, and obtained (red: main axis, blue: secondary axis, black: third axis).
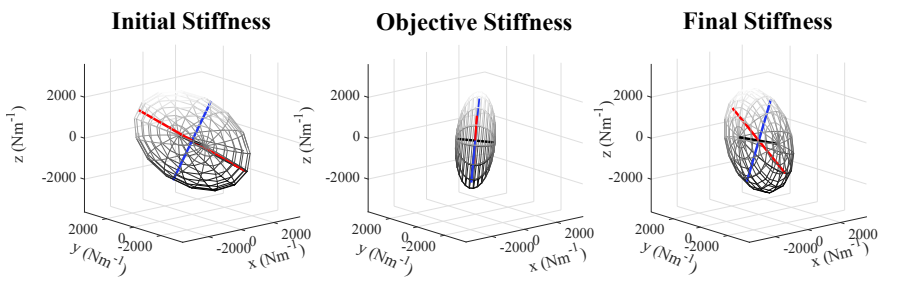

Fig. 4: Second apple experiment $\left(\operatorname{Rot}\left(Z, \frac{\pi}{4}\right)\right) . \mathbf{K}_{t}$ initial, targeted, and obtained (red: main axis, blue: secondary axis, black: third axis).

from a similar position to the final one of the first experiment (Fig. 3 and 5), and commanding the opposite transformation in the stiffness $\left(\operatorname{Rot}\left(Y, \frac{-\pi}{2}\right)\right)$, the hand converges to a final pose similar to the initial one of the first experiment.

With respect to the length ratio error $\beta$, as the new desired stiffness is obtained from the initial one, there is no initial error and $\beta$ is kept quite low for all trials. Regarding the joint stiffness parameter $\alpha$, for the first two experiments, the found value is lower than the initial, while for the last it reaches the maximum value. This can be correlated with the fact that for the first two trials, PCR value is increased, whereas it decreases for the third trial. This means that whereas the first two final configurations where quite stable, the last one had to use more power to stabilize the grasp. This was further verified during the real experiments, in which less stable contacts were clearly visible. Incrementing the maximum allowed joint stiffness could help to achieve a better grasp. However, due to the lack of sensory information, and the fact that the real object position can differ from the simulated one, this might not be enough. Allowing the hand fingers to target a deeper position
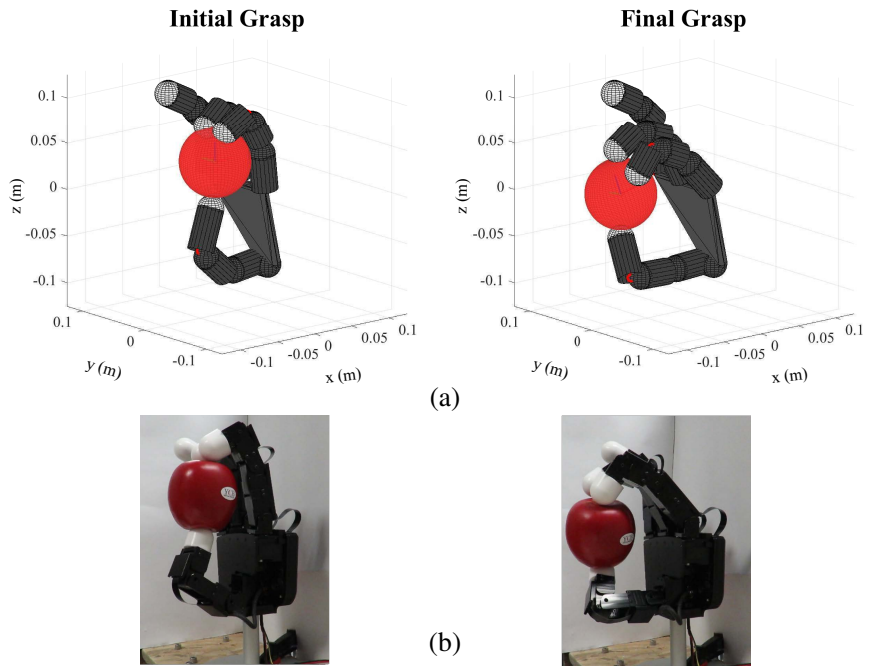

(a)

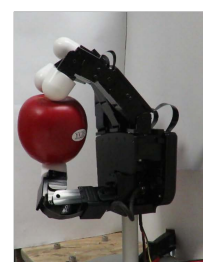

(b)

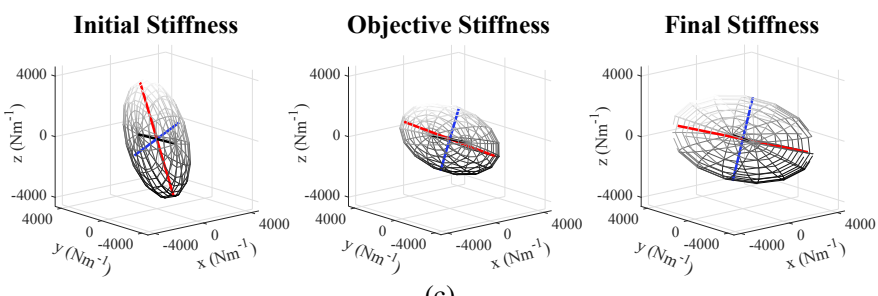

(c)

Fig. 5: Third apple experiment $\left(\operatorname{Rot}\left(Y, \frac{-\pi}{4}\right)\right)$. (a) Stabilized model of the Allegro Hand in the initial and final configurations (b) Allegro Hand in initial and final configurations (c) $\mathbf{K}_{t}$ initial, targeted, and obtained (red: main axis, blue: secondary axis, black: third axis).

inside the object would make the hand reach and contact better the object, though it could constraint the thumb movement in some directions.

Fig. 6, Fig. 7, and Fig. 8 show the results for the experimental trials with the cube, orange, and "crackers" pack respectively. In the stiffness plot, the desired stiffness axis has been magnified to be able to better appreciate the shape. Table II displays the corresponding numerical results.

TABLE II: Initial values and results of the algorithm when manipulating the cube, orange, and the "crakers" pack. $\theta$ error is expressed in $\operatorname{deg}, \beta$ and PCR are dimensionless, and $\alpha$ is $N \mathrm{~m} / \mathrm{rad}$. 'Init' stands for initial configuration.

\begin{tabular}{|c|c|c|c|c|c|}
\hline Cube & $\boldsymbol{\theta}_{\mathbf{1}}$ & $\boldsymbol{\theta}_{\mathbf{2}}$ & $\boldsymbol{\beta}$ & $\boldsymbol{\alpha}$ & PCR \\
\hline Init $\left(K_{d, x x}=2000\right)$ & 55.64 & 55.75 & 7.179 & 4 & 0.1676 \\
\hline Final $\left(K_{d, x x}=2000\right)$ & 23.25 & 24.84 & 7.0135 & 5.998 & 0.06668 \\
\hline Orange & $\boldsymbol{\theta}_{\mathbf{1}}$ & $\boldsymbol{\theta}_{\mathbf{2}}$ & $\boldsymbol{\beta}$ & $\boldsymbol{\alpha}$ & PCR \\
\hline Init $\left(K_{d, x x}=2000\right)$ & 58.71 & 59.87 & 6.1077 & 4 & 0.1346 \\
\hline Final $\left(K_{d, x x}=2000\right)$ & 21.72 & 22.61 & 6.24 & 6 & 0.1208 \\
\hline "Crackers" & $\boldsymbol{\theta}_{\mathbf{1}}$ & $\boldsymbol{\theta}_{\mathbf{2}}$ & $\boldsymbol{\beta}$ & $\boldsymbol{\alpha}$ & PCR \\
\hline Init $\left(K_{d, z z}=2000\right)$ & 38.55 & 39.5 & 8.345 & 4 & 0.1782 \\
\hline Final $\left(K_{d, z z}=2000\right)$ & 4.69 & 6.449 & 8.305 & 2.135 & 0.0777 \\
\hline
\end{tabular}

Again, errors in the main axes orientations are reduced, being this reduction maximum for the "crackers" pack test. Indeed, in this case the initial grasp configuration provided a nice starting stiffness towards the targeted one. Therefore, a smaller movement of the hand with respect to other trials is seen, though the error is significantly reduced. The length ratio error $\beta$ in these cases is not significantly modified during the optimization. This means that, as specified in (3), the optimization gives more weight to the orientation than to the 

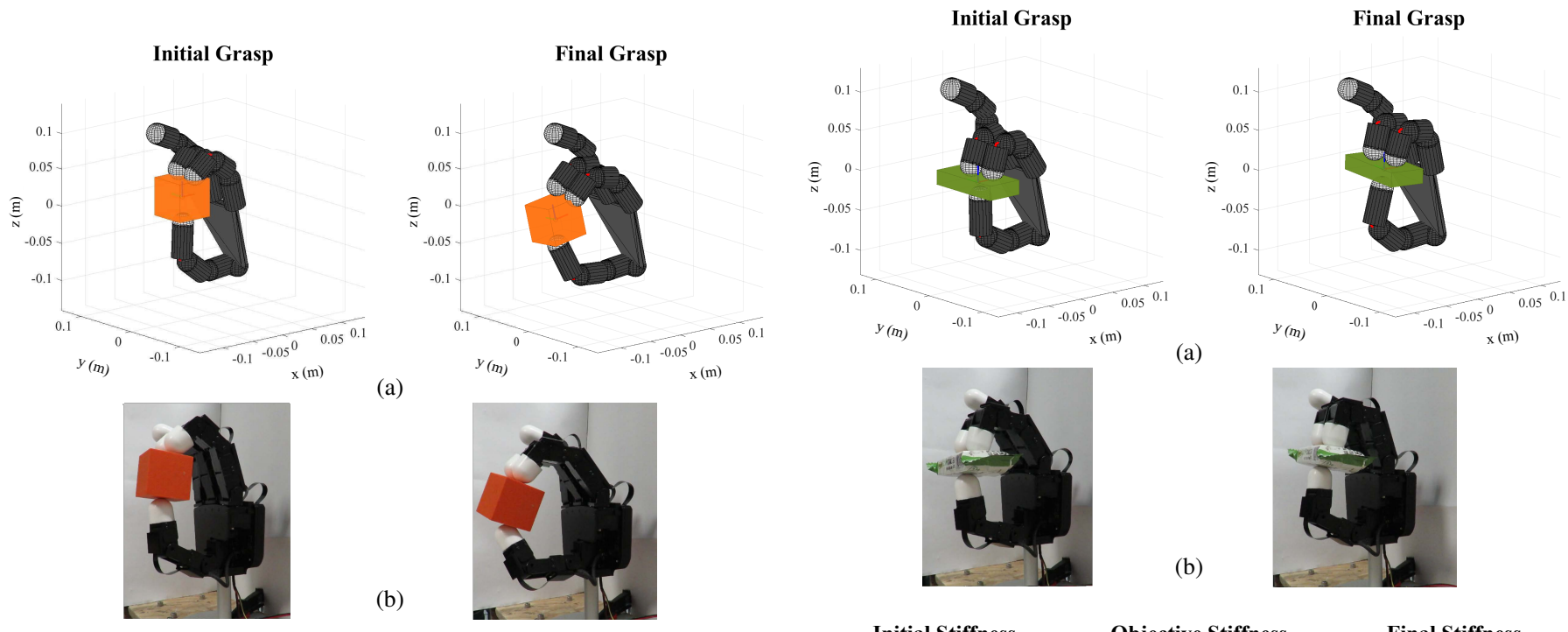

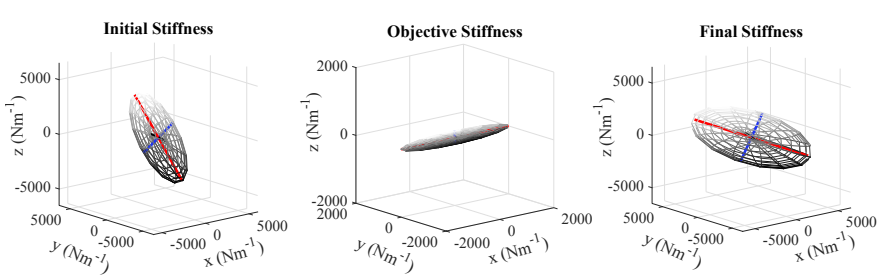

(c)

Fig. 6: Cube experiment $\left(K_{d, x x}=2000 \mathrm{~N} / \mathrm{m}\right)$. (a) Stabilized model of the Allegro Hand in the initial and final configurations (b) Allegro Hand in initial and final configurations (c) $\mathbf{K}_{t}$ initial, targeted, and obtained (red: main axis, blue: secondary axis, black: third axis).
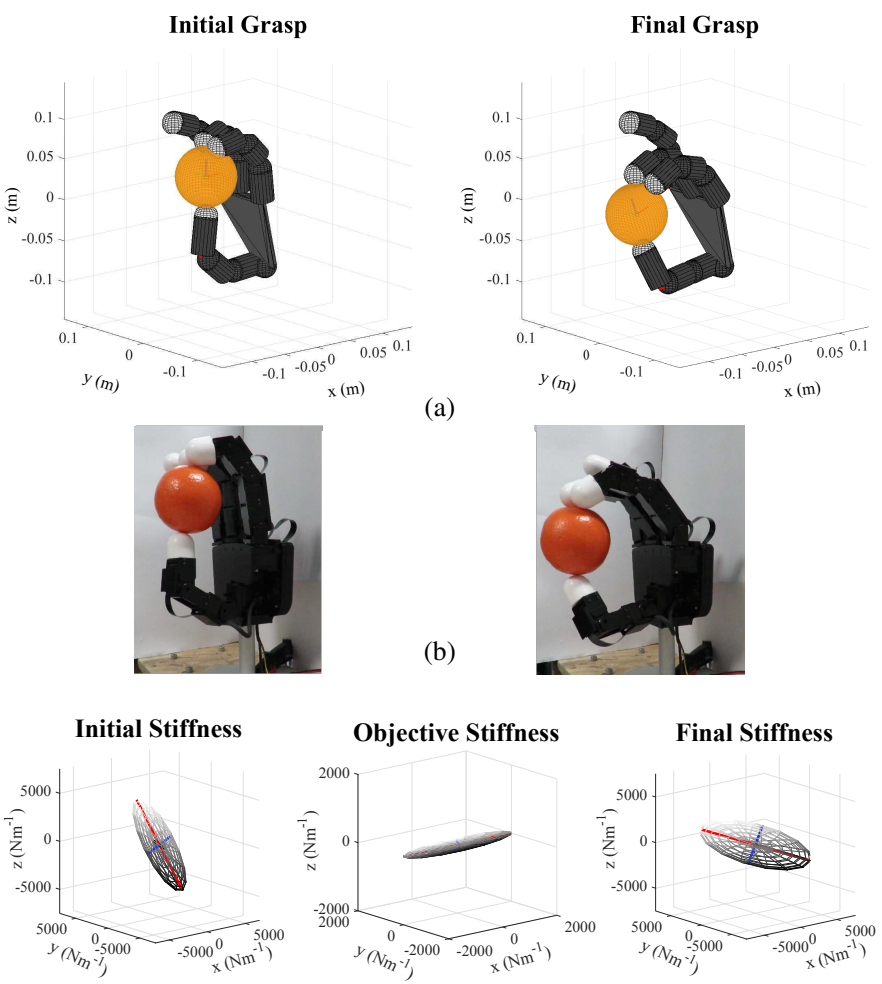

(c)

Fig. 7: Orange experiment $\left(K_{d, x x}=2000 \mathrm{~N} / \mathrm{m}\right)$. (a) Stabilized model of the Allegro Hand in the initial and final configurations (b) Allegro Hand in initial and final configurations (c) $\mathbf{K}_{t}$ initial, targeted, and obtained (red: main axis, blue: secondary axis, black: third axis).

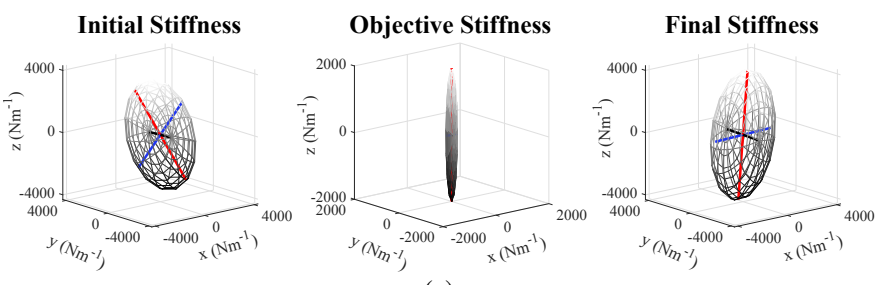

(c)

Fig. 8: "Crackers" pack experiment $\left(K_{d, z z}=2000 \mathrm{~N} / \mathrm{m}\right)$. (a) Stabilized model of the Allegro Hand in the initial and final configurations (b) Allegro Hand in initial and final configurations (c) $\mathbf{K}_{t}$ initial, targeted, and obtained (red: main axis, blue: secondary axis, black: third axis).

length ratio. Similarly, in these cases PCR decreases with respect to the initial configuration, meaning that the final grasps are less robust (though still stable). Specially for the cube and the "crackers" pack these values are lower. For the cube and the orange, a higher joint stiffness $\alpha$ is obtained. In this case, a problem is mainly detectable from the lack of sensory information, providing the location of the object center. Indeed, in Fig. 6 and Fig. 7, it can be observed how the virtual object model orientation in the final configuration and the real one are quite different. This results in modified contact points and contact forces.

Nonetheless, it must be noted that 20 repetitions were carried out for all objects and desired stiffness, and the hand never dropped the object. This means that the method was able to keep the grasp $100 \%$ of the times.

Regarding the experiments to test the usefulness of the stabilization procedure and the robustness term (PCR), previous results are compared to those of the 20 repetitions without the stability. For the latter, it could be observed that in the best case (cube), the hand lost the object $20 \%$ of the times. For the apple first case $\left(\operatorname{Rot}\left(Y, \frac{\pi}{4}\right)\right)$, the success rate decreased to only $10 \%$ of the cases. It further decreased for the apple last case $\left(\operatorname{Rot}\left(Y, \frac{-\pi}{4}\right)\right)$ and the "crackers" pack case, where the manipulation was successful only $5 \%$ of the times. The object fell in all the repetitions ( $0 \%$ success rate) for the second test case of the apple $\left(\operatorname{Rot}\left(Z, \frac{\pi}{4}\right)\right)$ and the orange. Representative trials with and without stability can be visualized at the end of the video [28].

In view of the presented results, it can be asserted that the proposed method is able to drive the initial grasp towards 
the desired grasp stiffness through the modification of the hand configuration (CDS) and joint stiffness (CMS). This is done without loosing grasp stability in all the process. Alternative experiments proved the usefulness of the stabilization procedure. Besides, results show that the optimization method, as expected from (3), gave more weight to the main stiffness directions, being those the parameters in which errors where reduced most. Additionally, these results proved that the method can be easily generalized to diverse object shapes. The lack of visual and force information was tackled by using additional optimization variables and geometrical calculations. These estimations proved to be sufficient to achieve the desired grasp configuration while keeping the stability.

\section{CONCLUSIONS}

This manuscript proposed an algorithm to achieve a desired grasp stiffness by modifying the hand configuration and joint stiffness while keeping a stable grasp. Changing the stiffness of a robotic grasp can be very useful in teleoperation tasks both to map the stiffness of the master onto the slave, and to adapt the grasp stiffness to target desired values depending on the set of tasks to be executed. With this aim, a set of high-level features of the grasp stiffness ellipsoid geometry were selected to define the main requirements of the task. The adjustment of the stiffness ellipsoid volume was left to mainly affect the stability and to be tuned, if requested, depending on external inputs. The preliminary experiments show that the method is able to approximate a desired stiffness shape generating a stable hand trajectory. This approximation is however not perfect due to the hardware configuration and limitations of the actual robotic hand.

Adding force-sensors or visual feedback would clearly enhance the method, with the drawback of an increased cost. The method was tested with two kind of object shapes (spheres and cuboids). In the lack of sensory information, the generalization to other shapes (like cylinders), would only require to analytically find the intersection between the fingertip sphere and the new shape. On the other hand, the method is designed so far to deal with fingertip grasps. Other kind of grasps would require the manual definition of the contact points on the fingers or the mentioned sensory information. Expanding the method to consider more finger contacts and grasp types is within the future work scope. Also, testing the algorithm with different robotic hands would provide valuable information regarding feasible grasp stiffness and influence of the hardware design.

\section{REFERENCES}

[1] M. Grebenstein, A. Albu-Schäffer, T. Bahls, M. Chalon, O. Eiberger, W. Friedl, R. Gruber, S. Haddadin, U. Hagn, R. Haslinger, et al., "The dlr hand arm system," in 2011 IEEE International Conference on Robotics and Automation, May 2011, pp. 3175-3182.

[2] A. Bicchi, "Hands for dextrous manipulation and robust grasping: a difficult road towards simplicity," IEEE Trans. on Robotics and Automation, vol. 16, no. 6, pp. 652-662, December 2000.

[3] T. Schlegl, M. Buss, T. Omata, and G. Schmidt, "Fast dextrous regrasping with optimal contact forces and contact sensor-based impedance control," in Proceedings 2001 ICRA. IEEE International Conference on Robotics and Automation, vol. 1, May 2001, pp. 103-108.

[4] M. R. Cutkosky and I. Kao, "Computing and controlling compliance of a robotic hand," IEEE Transactions on Robotics and Automation, vol. 5, no. 2, pp. 151-165, Apr 1989.
[5] A. M. Dollar and R. D. Howe, "Towards grasping in unstructured environments: grasper compliance and configuration optimization," Advanced Robotics, vol. 19, no. 5, pp. 523-543, 2005.

[6] F. Caccavale, P. Chiacchio, A. Marino, and L. Villani, "Six-dof impedance control of dual-arm cooperative manipulators," IEEE/ASME Transactions on Mechatronics, vol. 13, no. 5, pp. 576-586, Oct 2008.

[7] A. Ajoudani, S. B. Godfrey, M. Bianchi, M. G. Catalano, G. Grioli, N. Tsagarakis, and A. Bicchi, "Exploring teleimpedance and tactile feedback for intuitive control of the pisa/iit softhand," IEEE Transactions on Haptics, vol. 7, no. 2, pp. 203-215, April 2014.

[8] P. Rao, G. C. Thomas, L. Sentis, and A. D. Deshpande, "Analyzing achievable stiffness control bounds of robotic hands with compliantly coupled finger joints," in IEEE International Conference on Robotics and Automation (ICRA), May 2017.

[9] N. Bernstein, The Coordination and Regulation of Movements. Pergamon Press, Oxford, 1967.

[10] T. Flash and N. Hogan, "The coordination of arm movements: an experimentally confirmed mathematical model," Journal of neuroscience, vol. 5, no. 7, pp. 1688-1703, Jul 1985.

[11] M. Santello, M. Flanders, and J. F. Soechting, "Postural hand synergies for tool use," Journal of Neuroscience, vol. 18, no. 23, pp. $10105-$ 10115 , Dec 1998.

[12] G. Gioioso, G. Salvietti, M. Malvezzi, and D. Prattichizzo, "Mapping synergies from human to robotic hands with dissimilar kinematics: An approach in the object domain," IEEE Transactions on Robotics, vol. 29, no. 4, pp. 825-837, Aug 2013.

[13] M. Rossi, A. Altobelli, S. B. Godfrey, A. Ajoudani, and A. Bicchi, "Electromyographic mapping of finger stiffness in tripod grasp: a proof of concept," in 2015 IEEE International Conference on Rehabilitation Robotics (ICORR), Aug 2015, pp. 181-186.

[14] M. R. Cutkosky, "On grasp choice, grasp models, and the design of hands for manufacturing tasks," IEEE Transactions on Robotics and Automation, vol. 5, no. 3, pp. 269-279, Jun 1989.

[15] T. E. Milner and D. W. Franklin, "Characterization of multijoint finger stiffness: dependence on finger posture and force direction," IEEE Trans. on Biomed. Engineering, vol. 45, no. 11, pp. 1363-1375, Nov 1998.

[16] S.-F. Chen and I. Kao, "Conservative congruence transformation for joint and cartesian stiffness matrices of robotic hands and fingers," The Int. J. of Robotics Research, vol. 19, no. 9, pp. 835-847, Sep 2000.

[17] A. Ajoudani, C. Fang, N. Tsagarakis, and A. Bicchi, "Reducedcomplexity representation of the human arm active endpoint stiffness for supervisory control of remote manipulation," The International Journal of Robotics Research, vol. 37, no. 1, pp. 155-167, Jan 2018.

[18] A. Ajoudani, M. Gabiccini, N. G. Tsagarakis, and A. Bicchi, "Humanlike impedance and minimum effort control for natural and efficient manipulation," in 2013 IEEE International Conference on Robotics and Automation, May 2013, pp. 4499-4505.

[19] V. Ruiz Garate, N. Tsagarakis, A. Bicchi, and A. Ajoudani, "On the common-mode and configuration-dependent stiffness control of multiple degrees of freedom hands," in 2017 IEEE-RAS 17th International Conference on Humanoid Robotics, Nov 2017, pp. 113-120.

[20] W. Robotics, "Allegro hand," 2017, [accessed 12-September-2017]. [Online]. Available: http://wiki.wonikrobotics.com/AllegroHandWiki/ index.php/Allegro_Hand

[21] M. Malvezzi and D. Prattichizzo, "Evaluation of grasp stiffness in underactuated compliant hands," in 2013 IEEE International Conference on Robotics and Automation, May 2013, pp. 2074-2079.

[22] D. Prattichizzo and J. C. Trinkle, Grasping in the Springer Handbook of Robotics. Springer, July 2016.

[23] A. Bicchi, "On the problem of decomposing grasp and manipulation forces in multiple whole-limb manipulation," Robotics and Autonomous Systems, vol. 13, no. 2, pp. 127 - 147, Jul 1994.

[24] M. Pozzi, M. Malvezzi, and D. Prattichizzo, "On grasp quality measures: Grasp robustness and contact force distribution in underactuated and compliant robotic hands," IEEE Robotics and Automation Letters, vol. 2, no. 1, pp. 329-336, Jan 2017.

[25] M. Gabiccini, A. Bicchi, D. Prattichizzo, and M. Malvezzi, "On the role of hand synergies in the optimal choice of grasping forces," Autonomous Robots, vol. 31, no. 2, p. 235, Jul 2011.

[26] M. Malvezzi, G. Gioioso, G. Salvietti, and D. Prattichizzo, "Syngrasp: A matlab toolbox for underactuated and compliant hands," IEEE Robotics Automation Magazine, vol. 22, no. 4, pp. 52-68, Dec 2015.

[27] P. Corke, Robotics, Vision and Control: Fundamental Algorithms in MATLAB, ser. Springer Tracts in Advanced Robotics. Springer, 2011. [28] Video attachment, https://youtu.be/xeEaU8Da8iY. 